\title{
Algumas contribuições de von Neumann à Física Matemática
}

\author{
WALTER F. WRESZINSKI
}

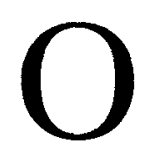

QUE CARACTERIZA as muitas contribuições de von Neumann à Física Matemática é a profundidade e extrema originalidade das suas idéias, que parecem surgir do nada. Cada uma delas abriu novos caminhos, e muitas conduziram a desenvolvimentos surpreendentes. Nesta palestra, quero concentrar-me em duas dessas idéias e seus desenvolvimentos subseqüentes: a teoria de perturbações, e a dos avoided crossings, com maior ênfase na última. Por completeza, menciono algumas das suas contribuições fundamentais, que não serão aqui abordadas: os métodos matemáticos da mecânica quântica (von Neumann, 1932) (inclusive a teoria dos operadores não limitados e grafos (id., 1929) e o teorema fundamental da unicidade relativo às relações canônicas de comutação (id., 1931); a teoria das álgebras de von Neumann (Dixmier, 1957) e suas aplicações à mecânica estatística (Ruelle, 1969); a teoria dos espaços produto-direto incompletos e suas aplicações, por exemplo, modelo de Dicke do MASER por Hepp \& Lieb (1973) e, finalmente, os métodos da teoria ergódica e suas aplicações à mecânica clássica (Koopman-von Neumann (1932). Cada um desses tópicos seria assunto para várias palestras. Por exemplo, o teorema da unicidade é específico a número finito de graus de liberdade: no caso de sistemas de número infinito de graus de liberdade (teoria de campos) não há unicidade, e sim variedade nãoenumerável de representações inequivalentes estranhas das relações de comutação, o que representa uma fonte de graves problemas intrínsecos dessa teoria.

O problema da teoria de perturbações é central em análise funcional, particurlarmente a do espectro singular: dado um operador auto-adjunto $H$ com espectro puramente pontual, e uma perturbação compacta $V$, quando é que $(H+V)$ tem também espectro puramente pontual? $\mathrm{O}$ início desse estudo foi o trabalho de von Newmann e Weyl, e o resultado do teorema de Weyl-von Newmann (1935) não é encorajador: qualquer 
operador auto-adjunto difere de um operador com espectro puramente pontual por um operador de norma Hilbert-Schmidt arbitrariamente pequena. Esse resultado gerou impressionante desenvolvimento: Kato e Ronsblum mostraram que perturbações da classe do traço preservam o espectro absolutamente contínuo (Kato, 1966), mas Carey \& Pincus (1976) mostraram que todo operador auto-adjunto sem parte absolutamente contínua difere de um operador com espectro pontual por um operador de norma traço arbitrariamente pequena. Pior ainda, o trabalho clássico de Donoghue (1965) dá exemplos de perturbações de posto um

$$
H(k)=H+K<. \varphi>\varphi
$$

em que

a) $H$ é puro ponto mas $H(k)$ é puro singular contínuo para $k \neq 0 \mathrm{e}$

b) $H$ é puro singular contínuo, mas $H(k)$ é puro ponto par $k \neq 0$.

A solução é considerar perturbações $V$, que não são pequenas em algum sentido abastrato, mas pequenas relativamente a $H$, no sentido de que elas não deslocam demasiadamente os autovetores de $H$. Grosseiramente, se $\left\{e_{n}\right\}$ é um conjunto ortonormal de autovetores do operador puro ponto $H$, uma condição é

$$
\Sigma_{2}\left\|V^{1 / 2} e_{n}\right\|<\infty
$$

Esse desenvolvimento é relativamente recente (Howland, 1987) mas mostra um aspecto fundamental do desenvolvimento das idéias de von Neumann. A condição acima é muito forte se comparada com os requisitos da teoria do espalhamento, refletindo o fato de que o espectro absolutamente contínuo é mais estável do que o espectro puramente pontual denso. Exemplos de espectro pontual denso hoje abundam em física matemática, na teoria de sistemas desordenados e quase-periódicos. Um exemplo é o modelo de Anderson de impurezas distribuídas aleatoriamente em um cristal, sobre o qual há diversos resultados recentes. Um deles, devido a Frölich e Spencer, garante que o espectro de operadores do tipo

$$
H=-\Delta+V_{\omega} \quad e m l^{2}\left(Z^{\nu}\right)
$$

onde $\left(V_{\omega} f\right)(x)=\omega(x) f(x)$, e $\{\omega(x)\}_{x} \varepsilon z^{\nu}$ são variáveis aleatórias independentes e identicamente distribuídas, e $(-\Delta)$ é o laplaciano de diferença e tem espectro puro ponto se a desordem for suficientemente elevada (Fröhlich \& Spencer, 1986). 
O segundo tópico que abordaremos diz respeito à teoria de avoided crossings devida a von Neumann e Wigner (1927). Essa teoria tornouse extremamente atual na última década, com implicações profundas na teoria de sistemas quânticos classicamente caóticos e da fase geométrica (fase de Berry). Referência a esses desenvolvimentos recentes encontra-se em Avron et al. (1989) e no extraordinário apêndice do livro de Arnold (1974). A idéia-chave é, entretanto, de von Neumann.

Considere uma matriz hermitiana $H(\phi)$, dependendo de um parâmetro $\phi \in E$ (o espaço de parâmetros, um subconjunto de $R^{n}$. Sejam $E_{\jmath}(\phi)$ os autovalores da matriz. Os cruzamentos (crossings) de autovalores têm papel fundamental em diversas teorias, por exemplo, em mecânica quântica, onde o teorema adiabático requer que $E_{\jmath}\left(\phi_{2}\right)$ seja um autovalor isolado. O operador de projeção correspondente é \&.

$$
P_{j}\left(\phi_{2}\right)=\frac{1}{2 \pi i} \int_{\gamma_{j}} \frac{d z}{H(\phi)-z}
$$

onde $\gamma_{j}$ é um contorno circundando o j-ésimo autovalor no espectro. $P_{j}(\phi)$, herda o caráter liso de $H(\phi)$ desde que $\gamma_{j}$ fique fora do espectro; quando as lacunas no espectro se fecham, o caráter liso pode desaparecer. Seja $D(P)$ o conjunto dos pontos de $E$ onde $P$ não é liso, ou seja, é o conjunto de pontos de cruzamentos de níveis.

Considere o comportamento local de $E_{j}(\phi)$ e $P_{j}(\phi)$ próximo a um cruzamento de dois níveis em $\phi$. Restringindo o hamiltoniano ao subespaço degenerado em $\phi$ fornece uma matriz hermitiana $2 \times 2$ :

$$
\begin{aligned}
h(\phi) & =\left[\begin{array}{cc}
\langle\psi|H(\phi)| \psi\rangle & \langle\psi|H(\phi)| \varphi\rangle \\
\langle\psi|H(\phi)| \psi\rangle & \langle\psi|H(\phi)| \varphi\rangle
\end{array}\right] \\
& \equiv \epsilon_{0}(\phi) 1+\vec{\epsilon}(\phi) . \vec{\sigma}
\end{aligned}
$$

onde $|\psi\rangle \mathrm{e} / \varphi\rangle$ são os dois autovetores independentes de $H(\phi)$ em $\phi$. Aqui, $\vec{\epsilon}(\phi)$ é uma função em $R^{3}$, e $\vec{\sigma}$ o tripleto de matrizes de Pauli. Os dois autovalores da matriz $h(\phi)$ são

$$
E_{ \pm}(\phi)=\epsilon_{0}(\phi) \pm|\vec{\epsilon}(\phi)|
$$

onde $\vec{\epsilon}(\phi)=\overrightarrow{0}$. Assim, para matrizes hermitianas complexas $D(P)$ tem co-dimensão 3; para matrizes reais, a co-dimensão é 2 . As autoprojeções são:

$$
P_{ \pm}(\phi)=[\dot{1} \pm \hat{\epsilon}(\phi) . \vec{\sigma}] / 2
$$


onde $\hat{\epsilon}$ é o vetor unitário associado à $\vec{\epsilon}$. Devido ao valor absoluto em (1) e à normalização a vetores unitários em (2), nem $E_{ \pm}(\phi)$ nem $P_{ \pm}(\phi)$ são necessariamente lisos em $\phi$. No espaço $\vec{\epsilon},(1)$ descreve uma cônica:

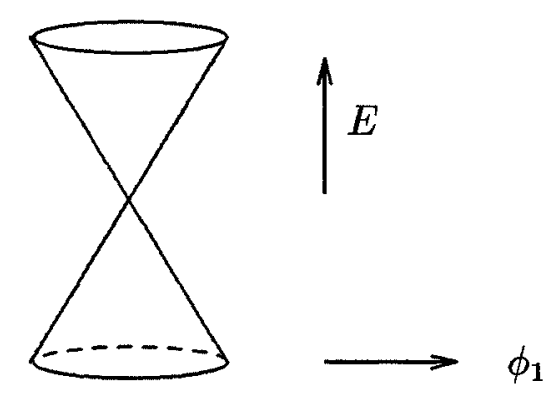

Berry chama tais pontos de diabólicos. Os autovalores são contínuos em $\vec{\epsilon}$ mas não-lisos, e as projeções não são nem mesmo contínuas próximas de $\vec{\epsilon}=\overrightarrow{0}$.

A idéia fundamental de von Neumann, totalmente original, é a seguinte, fornecendo uma prova alternativa do teorema. $O$ espaço de matrizes hermitianas $n \times n$ é um espaço vetorial de $n^{2}$ dimensões. Mostremos inicialmente que o espaço de matrizes hermitianas não-degeneradas é de dimensão plena $\left(n^{2}\right)$. A matriz unitária que diagonaliza uma dada matriz hermitiana com espectro não-degenerado fixo

$$
E_{1}<E_{2}<\ldots \quad<E_{n}
$$

está determinada a menos de uma matriz diagonal unitária. Dessa forma, existe uma correspondência biunívoca entre matrizes hermitianas nãodegeneradas com espectro fixo e elementos de

$$
U(n) /[U(1)]^{n}
$$

Como $\operatorname{dim}[U(n)]=n^{2}$, o espaço em (4) é $n(n-1)$ dimensional, o que, junto com as n dimensões associadas à variação dos $E_{j}(3)$, fornece $n^{2}$, a dimensão plena.

Considere, agora, as matrizes hermitianas com um estado degenerado, digamos o fundamental. A equação (3) é substituída por

$$
E_{1}=E_{2} \ldots \ldots . .<E_{n}
$$

As unitárias diagonalizantes correspondentes são identificadas com os elementos de

$$
U(n) /\left[[U(1)]^{n-2} \times U(2)\right]
$$


que é de dimensão $n^{2}-(n-2)-4=n(n-1)-2$. A dimensão do espaço associado à variação dos $E_{j}$ em (5) é $n-1$ e a dimensão total do espaço com uma degenerescência é $n(n-1)-2+n-1=n^{2}-3$. A codimensão é 3 e é independente de $n$. De forma mais geral, o espaço das matrizes hermitianas com $m$-degenerescências tem codimensão

$$
(m-1)+\operatorname{dim} U(m)-m \operatorname{dim} U(1)=m^{2}-1
$$

As codimensões são independentes da dimensão das matrizes e valem para operadores que são limites de matrizes e tem espectro discreto.

O teorema de von Neumann-Wigner nesta forma tem conteúdo essencialmente geométrico, indicando que o conjunto de elipsóides de revolução é uma união finita de subvariedades diferenciáveis de codimensão $\geq 2$ na variedade de todos os elipsóides de revolução. Esse é o ponto de vista de Arnold (1974). Essa idéia foi fecunda.

O teorema sugere que uma família de operadores dependendo de $n$ parâmetros tem cruzamentos de níveis em um conjunto de codimensão 3 no espaço dos parâmetros. Isto é um ansatz, não um teorema, porque a família a $n$-parâmetros pode estar imersa em uma forma especial no espaço de todas as matrizes hermitianas: considere a equação de Schrödinger na reta com potencial $V(x ; \phi)$ dependendo de $n$ parâmetros $\phi \equiv\left(\phi_{1}, \ldots, \phi_{n}\right)$ com $V \rightarrow \infty$ para $|x| \rightarrow \infty$. O ansatz de von NeumannWigner fornece codimensão 2 neste caso, mas falha arbitrariamente mal porque o espectro é simples devido a uma identidade wronskiana.

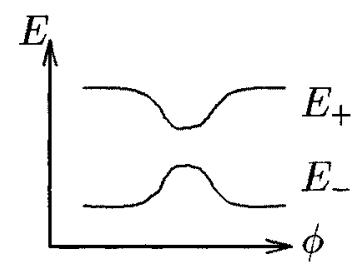

Entretanto o ansatz funciona em geral extraordinariamente bem para sistemas quânticos genéricos (não classicamente integráveis), com hamiltonianas reais; variando-se apenas um parâmetro, não há cruzamentos e $\operatorname{sim}$ avoided crossings, como os da figura mencionada. Esse fato tem implicaçôes profundas na moderna teoria de sistemas quânticos classicamente caóticos; a estatística de níveis revela que a dinâmica clássica deixa traços, correspondentes a uma repulsão de niveis (Berry, 1983). 
Gostaríamos de mencionar ainda um importante problema intimamente ligado à filosofia de von Neumann-Wigner: o da fase geométrica ou fase de Berry.

A fase de Berry é um conceito geométrico ligado ao transporte paralelo em uma superfície curva. Se um vetor for deslocado paralelamente ao longo de uma curva fechada, por exemplo, sobre a superfície de uma esfera, ele formará no final com a direção inicial um ângulo característico da curvatura da superfície.

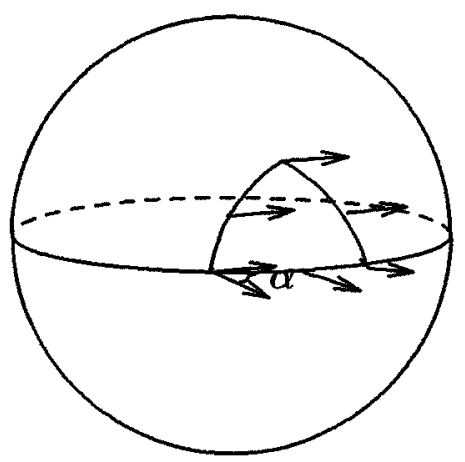

Em analogia à superfície, consideramos os estados (puros) em mecânica quântica. Esses estados são descritos por raios (duas funções de onda pertencem ao mesmo raio quando elas se diferenciam apenas por uma fase). As fases das funções de onda correspondem às direções dos vetores sobre a superfície. $O$ transporte paralelo no espaço de estados da mecânica quântica é dado pela equação adiabática (a seguir). Quando, então, uma fase for transportada paralelamente ao longo de uma curva fechada no espaço de estados da mecânica quântica, ela pode no final ser distinta do valor inicial. A diferença entre os valores final e inicial denomina-se fase de Berry.

Para introduzir esse conceito em maior detalhe, consideremos o exemplo de um spin em mecânica quântica. Neste caso, como vimos, é importante distinguir entre a função de onda, isto é, o vetor de spin

$$
\begin{gathered}
\psi(\alpha, \beta)=\alpha|\uparrow>+\beta| \downarrow>\quad|\alpha|^{2}+|\beta|^{2}=1 \\
\left|\uparrow>=\left(\begin{array}{l}
1 \\
0
\end{array}\right) \quad\right| \downarrow>=\left(\begin{array}{l}
0 \\
1
\end{array}\right)
\end{gathered}
$$

e o raio correspondente. Em vez de raios pode-se freqüentemente utilizar os projetores nos vetores de spin, que são matrizes $2 \times 2$, e podem ser 
parametrizadas por uma superfície esférica em três dimensões:

$$
P(\vec{n})=\frac{1}{2}(1+\vec{n} \vec{\sigma}) \quad|\vec{n}|=1
$$

onde $\vec{\sigma}$ denota o vetor das três matrizes de Pauli. Assim, os estados quânticos de um spin podem ser identificados com uma superfície esférica bidimensional. Sobre ela consideramos a curva fechada $\omega=\vec{n}(s) / 0 \leq s \leq$ $1, \vec{n}(0)=\vec{n}(1)$ e tentamos encontrar vetores de spin normalizados tais que

$$
P(\vec{n}(s))=/ \psi(s)><\psi(s) /
$$

A dificuldade aí reside na escolha da fase, já que o vetor de spin só pode ser reconstruído do projetor a menos de uma fase. O seguinte requisito torna única a escolha da fase, dado $\psi(0)$ :

$$
\langle\psi(s), \dot{\psi}(s)\rangle=0 \quad 0 \leq s \leq 1
$$

Esta é a equação adiabática (Simon, 1983; Kato, 1950) que é sempre verdadeira para $\psi$ real e representa uma prescrição de como a fase dada para $s=0$ deve ser transportada paralelamente ao longo da curva fechada. Ela tem uma característica comum com o transporte paralelo de vetores: que a variação $\delta \psi$ seja ortogonal a $\psi$; como apenas um número real - a fase - é procurado, essa condição é suficiente. Da mesma forma, como sabemos, do transporte paralelo de vetores sobre a esfera surge uma diferença de fase entre $\psi(1)$ e $\psi(0)$, a fase de Berry $\Gamma$ :

$$
\psi(1)=e^{2 \pi i \Gamma} \psi(0)
$$

Uma curva no espaço de estados da mecânica quântica pode ser gerada da seguinte forma: Consideremos por exemplo, a matriz hamiltoniana

$$
H(\vec{B})=\frac{1}{2} \vec{B} \cdot \vec{\sigma}
$$

de um spin $1 / 2$ em um campo magnético $\vec{B}$. O estado quântico correspondente ao autovalor $|\vec{B}| / 2$ é o projetor $P(\vec{n}=\vec{B} /|\vec{B}|)$. $\vec{B}$ tem o papel de um parâmetro, o espaço de parâmetros é $R^{3}\{0\}$ que é isomorfo à esfera (a exclusão do 0 é devida ao fato de que este é o ponto de cruzamento dos autovalores, e o teorema adiabático não vale para esses pontos). Assim, uma curva no espaço dos parâmetros gera uma outra no espaço de estado quântico.

A fase de Berry pode ser expressa apenas através dos estados (projetores) - as fases das funções de onda correspondentes não são necessárias, 
como veremos agora. Seja $\phi(\vec{B})$ uma escolha arbitrária de autovetores normalizados de $H(\vec{B})$ com autovalor $|\vec{B}| e \vec{B}(s) / 0 \leq s \leq 1$ uma curva fechada no espaço de parâmetros. Os vetores de spin $\psi(s)$ e $\phi(\vec{B}(s))-$ ambos autovetores com o mesmo autovalor diferenciam-se por construção apenas por uma fase

$$
\psi(s)=e^{2 \pi i \gamma(s)} \quad \phi(\vec{B}(s)) .
$$

Da equação adiabática segue então

$$
2 \pi i \dot{\gamma}(s)+\dot{\vec{B}}(s) \cdot \vec{A}(\vec{B}(s))=0
$$

onde

$$
\vec{A}(\vec{B})=\left\langle\phi(\vec{B}), \nabla_{\vec{B}} \phi(\vec{B})\right\rangle
$$

é um tipo de potencial vetor (conexão). Por integração e aplicação do teorema de Stokes

$$
\Gamma=\Upsilon(s=1)=\frac{i}{2 \pi} \int_{\omega} \vec{A} \vec{d} \vec{l}=\frac{i}{2 \pi} \int_{F} \nabla \times \vec{A} \cdot d \vec{\sigma}
$$

onde $\mathrm{F}$ é uma superfície cuja fronteira é $\omega$ no espaço $R^{3}\{0\}$. Usando a linguagem de formas diferenciais, isto é, exprimindo $\nabla_{\vec{B}} \phi \rightarrow d \phi$.

$$
\begin{gathered}
\Gamma=\frac{i}{2 p i} \int_{F}\langle d \phi, d \phi\rangle=\frac{i}{2 p i} \int_{F} V \\
V \equiv\langle d \phi, d \phi\rangle=\sum_{i<j} I m\left\langle\frac{\partial \phi}{\partial B_{i}}, \frac{\partial \phi}{\partial B_{j}}\right\rangle d B_{i} \bigwedge d B_{j}
\end{gathered}
$$

em termos de coordenadas locais. Tal fórmula não mostra a independência de $\Gamma$ de mudanças de fase de $\phi$ mas, por

$$
(\nabla \times \vec{A})_{r}=i \sum_{s, t} \epsilon_{r s t} t r P \frac{\partial P}{\partial B_{s}} \frac{\partial P}{\partial B_{t}}
$$

pode-se demonstrar independência.

A equação para $V$ mostra que $V=0$ se pudermos escolher $\phi(\vec{B})$ todos simultaneamente reais. Assim, o fenômeno da fase de Berry só está presente, e.g., em campos magnéticos, onde é impossível, porque o hamiltoniano não é invariante por inversão temporal. Consideremos agora o cálculo de $V(\vec{B})$. Por invariança rotacional, basta calcular $V$ em 
$\vec{B} \equiv(0.0 . B)$. Seja $\mid m>$ o autovetor com $\frac{1}{2} \sigma_{z}|m>=m| m>\left(m= \pm \frac{1}{2}\right)$, então para $\vec{B}$ próximo a $(0,0, B)$, podemos tomar

$$
\phi(\vec{B})=\exp \left[i\left(\frac{B_{x}}{B} \frac{\sigma_{y}}{2}-\frac{B_{y}}{B} \frac{\sigma_{x}}{2}\right)+0\left(B_{x}^{2}+B_{y}^{2}\right)\right] \mid m>
$$

de onde

$$
\begin{aligned}
d \phi & =i B^{-1}\left(d B_{x} \frac{\sigma}{2} y-d B_{y} \frac{\sigma}{2} x\right) \mid m> \\
<d \phi, d \phi> & =-B^{-2} d B_{x} \bigwedge d B_{y}<m\left|\left[\frac{\sigma}{2} y, \frac{\sigma}{2} x\right]\right| m> \\
& =i B^{-2} m d B_{x} \bigwedge d B_{y}
\end{aligned}
$$

e voltando a $\vec{B}$ geral

$$
V(\vec{B})=i m|\vec{B}|^{-2} A(\vec{B})
$$

onde $\mathrm{A}$ é a $<$ forma da área na esfera de raio $|\vec{B}|$; segue que

$$
\Gamma=\frac{i}{2 \pi} i m|\vec{B}|^{-2} A(\vec{B})=-\frac{M}{2 \pi} \Omega
$$

onde $\Omega$ é o ângulo sólido subentendido pela curva fechada que corresponde à órbita no espaço dos parâmetros. Em particular, se F for uma esfera (ou qualquer superfície da qual a origem é ponto interno, já que $d V=0$ )

$$
\Gamma=-2 m
$$

que é um inteiro. Isto não é coincidência: se $\omega$ for uma curva anti-horária ao longo do equador na esfera $F$, que a divide em dois hemisférios $F_{ \pm}$,

$$
\exp (2 \pi i \Gamma)=\exp \left(-\int_{F}+V\right)=\exp \left(\int_{F_{-}} V\right)
$$

$\log 0$

$$
\int_{F} V=2 \pi i \times(\text { inteiro }) \text {. }
$$

Esta é a bem conhecida quantização da integral da curvatura da classe de Chern do fibrado (que no caso é dado da seguinte forma: para cada $\vec{B}$ seja $\left.X_{|\vec{B}|}\right)$ o autoespaço de $H(\vec{B})$ correspondente ao autovalor $\left.\mid \vec{B} !\right)$.

Finalmente, qual é a ligação com a teoria de von Neumann-Wigner?

Considere duas autofunções reais $\psi_{ \pm}$de um hamiltoniano, dependente de parâmetros que designaremos por $\vec{B}$, cujas energias degeneram 
em um ponto do espaço dos parâmetros, digamos $\vec{B}=\overrightarrow{0}$ (sem perda de generalidade): quando tomadas ao longo de uma curva fechada $\gamma$ no espaço dos parâmetros $\psi_{+}$e $\psi_{-}$mudarão de sinal se e somente se $\gamma$ envolver uma degenerescência. Esse teorema é mencionado em Arnold (1974). Ele permite distinguir na prática que realmente existe uma degenerescência em $\vec{B}=\overrightarrow{0}$ e não apenas em quase tocar de cones, análogo ao da figura dos avoided crossings. É bem sabido que as funções de onda são univalentes com respeito às suas variáveis (e.g. $\vec{x}$ ), mas esse teorema mostra que, surpreendentemente, elas não precisam sê-lo com respeito aos parâmetros do hamiltoniano. Para provar esse resultado, note que próximo a $\vec{B}=0$, onde $E_{ \pm}(\overrightarrow{0})=0$, o hamiltoniano pode ser representado por

$$
H(\vec{B})=\vec{\sigma} \cdot \vec{B}
$$

desde que a degenerescência seja levantada em primeira ordem, que é o caso genérico. Se a matriz é real, a componente $\sigma_{y}$ é nula e ficamos com

$$
H(\vec{B})=\left(\begin{array}{cc}
B_{z} & B_{x} \\
B_{x} & -B_{z}
\end{array}\right)
$$

Neste caso, os níveis de energia se interceptam cônicamente no espaço $E, B_{x}, B_{z}$ (de fato, $\left.E_{+}(\vec{B})=-E_{-}(\vec{B})=\left(B_{x}^{2}+B_{z}^{2}\right)^{1 / 2}\right)$ e a fase de Berry é novamente

$$
\Gamma=-\frac{m}{2 \pi} \Omega
$$

onde $\Omega=2 \pi$ se $\gamma$ envolve a degenerescência $(\vec{B}=\overrightarrow{0})$ no sentido antihorário, zero de outra forma (é o ângulo sólido no plano $B_{y}=0$ ). Assim,

$$
\Gamma_{ \pm}=\mp \frac{1}{2 \pi} \times \frac{1}{2} 2 \pi=\mp \frac{1}{2}
$$

e a mudança de fase é $\exp \left(2 \pi i \Gamma_{\mp}\right)=\exp (\mp i \pi)=-1$. Como exemplo explícito, considere a curva

$$
\vec{B}(s)=B(\cos 2 \pi S, 0, \sin 2 \pi S) ; 0 \leq s \leq 1
$$

A autofunção de spin correspondente ao autovalor $+|\vec{B}(s)| / 2\left(\psi_{+}(s)\right)$ bem conhecida

$$
\psi_{+}(s)=\left[\begin{array}{c}
\sin (\pi s+\pi / 4) \\
\sin (\pi s-\pi / 4
\end{array}\right]
$$

e safisfaz à equação adiabática. Assim,

$$
\psi_{+}(0)=-\psi_{+}(1)
$$


isto é, a fase de Berry é $1 / 2$.

Para concluir: o teorema adiabático está demonstrado no trabalho clássico de Kato (1950); uma exposição simples do resultado encontra-se em Simon (1983).

Como vimos, as idéias de von Neummann no capítulo dos crossings geraram desenvolvimentos espantosos, com implicações profundas em geometria e em diversos campos da física matemática.

Referências bibliográficas

AVRON, J. E.; SADUN, L.; SEGERT, J. \& SIMON, B. Chern numbers, quaternions and Berry phases in Fermi systems. Coomm. math. Phys., v. 124, n. $595,1989$.

ARNOLD, V. Methods mathematiques de la mécanique classique. Mir, 1974 [apêndice 10].

BERRY, M. V. Chaotic behaviour of deterministic systems. Les Houches XXXVI, North Holland, 1983.

CAREY, R. W. \& PINCUS, J. D. Amer. J. Math., v. 98, n. 481, 1976.

DIXMIER, J. Les algèbres d'operateurs dans l'espace hilbertien. Algèbres de von Neumann, 1957.

DONOGHUE, W. Comm. Pure Appl. Math., v. 18, n. 559, 1965.

FRÖHLICH, J. \& SPENCER, T. Comm. Math. Phys., v. 88, n. 151, 1983.

HEPP, K. \& LIEB, E.H. Ann. Phys., v. 76, n. 360, 1973.

HOWLAND, J. S. J. Funct. Anal., v. 74, n. 52, 1987.

KATO, T. Perturbation theory for linear operators. Springer, 1966.

—. J. Phys. Soc. Japan, v. 5, n. 435, 1950.

RUELLE, D. Statistical mechanics. Gouthier, Villars Benjamin, 1969. 
SIMON, B. Phys. Rev. Lett., v. 51, n. 2167, 1983.

SIMON, B. \& WOLFF, T. Comm. Pure Appl. Math, v. 39, n. 75, 1986.

VON NEUMANN, J. Mathematische Grundlagen der quanten'mechnik. N.Y., Springer, 1932.

Allgemeine Eigenwerttheorie Hwemitescher Funktionalopeeratoren. Math. Ann., v. 102, n. 49, 1929.

Über adjungierte Funktionaloperatoren. Math. Ann., v. 2, n. 33, p. $294,1929$.

Die Eindeutigkeit der Schrödingerschen Operatores. Math. Ann., v. 104, n. $570,1931$.

Charakterisierung des Spektrums eines Integraloperators. Actualites Sci. et Ind., v. 229, n. 38, 1935.

VON NEUMANN, J. \& KOOOPMAN, B. O. Dynamical systems of continuous spectra. Proc. Nat. Acad. Sci., v. 18, n. 255, 1932.

VON .NEUMANN, J. \& WIGNER, E. P. Phys. Zeit., v. 30, n. 467, 1927.

Walter Wreszinski é professor do Departamento de Física Matemática do Instituto de Física da USP.

Palestra feita pelo autor no encontro A obra e o legado de John von Neumann, organizado pelo Instituto de Estudos Avançados da USP e peJa Academia Brasilejra de Ciências no Instituto de Matemática e Estatística da USP em 14 de novembro de 1995 . 